\title{
I Im Dickicht des geltenden Namensrechts
}

Das Namensrecht ist in den vergangenen sechzig Jahren zu einem für Außenstehende auf Anhieb kaum zu überblickenden „Normengewirr“3 angewachsen. Genügten der Urfassung des Bürgerlichen Gesetzbuchs noch wenige schlanke Paragraphen, um die Bürger mit einem Namen auszustatten, haben die späteren Gesetzgeber in zahlreichen Reformen das Recht der Personennamen erheblich aufgebläht.

Die Vorschriften zum Geburtsnamen (§§ 1616 ff. BGB), Ehe- und Begleitnamen (§ 1355 BGB) regeln die auf den ersten Blick recht triviale Frage nach dem Familiennamen eines Menschen mit großer Liebe zum Detail. Welchen verheirateten Bürgerinnen und Bürgern ist etwa bewusst, dass sie als Ehegatten nach der Eheschließung - auch wenn sie ihren Namen als gemeinsamen Ehenamen durchgesetzt haben - neben diesem Ehenamen als geführtem Familiennamen weiter ihren gleichlautenden Geburtsnamen behalten und es zu einer „verdeckten Namensänderung “ ${ }^{4}$ kommt. So trage ich z. B. - weil meine Ehefrau und ich vor vielen Jahren den Ehenamen „Dutta“ gewählt haben - rechtlich den Namen „Dutta geborener Dutta“. Dieser gleichlautende Geburtsname kann später sogar ein Eigenleben entwickeln. Nur ein Beispiel von vielen: Wird ein Ehegatte, dessen Geburtsname Ehename geworden ist, adoptiert, erhält dieser Ehegatte als Geburtsnamen den Namen des Annehmenden (§ 1757 Abs. 1 S. 1, § 1767 Abs. 2 BGB). Dieser Erwerb vollzieht sich zwar zunächst recht unauffällig, wenn sich diese Namensänderung nicht auf den Ehenamen erstreckt, weil der andere Ehegatte sich dieser nicht anschließt ( 1767 Abs. 2 S. 3 BGB). Der Namenserwerb kraft Adoption besitzt aber durchaus Relevanz. Der Ehegatte kann etwa nach einer Beendigung der Ehe zu seinem Geburtsnamen zurückkehren (§ 1355 Abs. 5 S. 2 Fall 1 BGB) und nun den durch Adoption erlangten „schlafenden“ Geburtsnamen wählen (§ 1355 Abs. 6 BGB) - aber auch zu dem ursprünglichen Geburtsnamen als zum Zeitpunkt der Bestimmung des Ehenamens „geführten“ Namen zurückkehren (§ 1355 Abs. 5 S. 2 Fall 2 BGB).

3 Fritz Sturm Europäisches Namensrecht im dritten Jahrtausend - Ein Blick in die Zukunft, in: FS für Dieter Henrich (2000) S. 611 (618); von „einem Zustand heillosen Wirrwarrs“ spricht Paul Heinrich Neuhaus Die Kinder der neuen „Familien“, ZfJ 1981, 37 (42).

$4 \mathrm{Zu}$ diesem Phänomen ausführlich Berthold Gaaz Verdeckte Namensänderungen, StAZ 2000, 357.

2 OpenAccess. @ 2020 Juristische Gesellschaft zu Berlin, publiziert von De Gruyter.

(c) BY-NC-ND Dieses Werk ist lizenziert unter der Creative Commons Attribution-NonCommercial-NoDerivatives 4.0 License. https://doi.org/10.1515/9783110709872-002 
Allenfalls bei den Regeln zum Vornamen seiner Bürger hält sich der Gesetzgeber bedeckt, ${ }^{5}$ jedenfalls bis vor Kurzem. Das Gesetz schweigt weitgehend zur Bestimmung des Vornamens durch die Eltern als Ausübung ihrer Personensorge ${ }^{6}$ und überlässt dieses Thema der Rechtsprechung, die mittlerweile eine liberale Position einnimmt, was die Zulässigkeit von Vornamen anbelangt. Insbesondere sind - auch aus verfassungsrechtlichen Gründen, auf die ich später noch eingehen muss (unten IV. 3.) - die Zeiten vorbei, in denen der von den Eltern gewählte Vorname gebräuchlich sein muss. ${ }^{7}$ „Saphina-Amber“ oder „Coco-Phelia“ - Namen aus der Umgebung meiner eigenen Kinder - lassen heute weder den Puls eines Standesbeamten noch den eines spielplatzgestählten Vaters in die Höhe schnellen. Der elterlichen Kreativität setzt nur das Kindeswohl eine Grenze: Die Eltern dürfen mit ihrer Vornamenswahl weder Befremden noch Anstoß erregen, das Kind weder der Lächerlichkeit preisgeben noch in der Entfaltung seiner Persönlichkeit beeinträchtigen. ${ }^{8}$ Wo die Grenze zum kindeswohlwidrigen Vornamen verläuft, ist freilich oftmals schwer zu bestimmen, wie sich vor ein paar Jahren auch hier im Kammergericht zeigte. Der 1. Zivilsenat sah den Namen „Djehad“ als kindeswohlgemäß an. ${ }^{9}$ Einerseits ist dieser Name (,für Gott kämpfen“) in der arabischen Welt nicht vollkommen unüblich, ähnlich wie bei uns der Name „Gotthilf“ - der Senat beruft sich in seiner Entscheidung zur Zulässigkeit dieses Namens daher auch auf „muslimische Rechtsgelehrte“. Andererseits werden die Assoziationen, die der Name „Djehad“ hervorruft, in einem europäisch geprägten Milieu wohl überwiegend nicht an dessen Semantik orientiert sein. Zunächst kann für die Zulässigkeit eines Vornamens nur die spontane Reaktion des sozialen Umfelds maßgeblich sein; es ist dem Kind als Namensträger nicht zumutbar, seinen Namen stets mit sprachwissenschaftlichen Ausführungen zu erklären. ${ }^{10}$ Die meisten Zeitgenossen in unserer Umgebung werden bei „Djehad“ spontan an das Konzept des „Heiligen Krieges“ denken, auch wenn es sich hierbei um eine falsche Übersetzung des Namens handeln sollte. Und diese Assoziation kann das Kind durchaus der Lächerlichkeit preisgeben. Stellen Sie sich nur für

5 Wobei in der Vergangenheit immer wieder eine gesetzliche Regelung der Vornamensbestimmung gefordert wurde, etwa von Uwe Diederichsen Vornamensgebung als Aufgabe für den Gesetzgeber, in: FS für Dieter Henrich (2000) S. 101; siehe auch den Vorschlag von Nicole Arndt Die Geschichte und Entwicklung des familienrechtlichen Namensrechts in Deutschland unter Berücksichtigung des Vornamensrechts (2003) S. $221 \mathrm{ff}$.

6 Etwa Reinhard Hepting/Anatol Dutta Familie und Personenstand (3. Aufl. 2019) Rn. IV-338 ff.

7 Hepting/Dutta (vorige Fn.) Rn. IV-373 ff.

8 Hepting/Dutta (Fn. 6) Rn. IV-358 ff.

9 KG 30.6.2009, StAZ 2009, 271 (272).

10 Hepting/Dutta (Fn. 6) Rn. IV-386; zu Recht kritisch auch Fabian Wall Darf ein Kind in Deutschland „Djehad“ heißen? NJOZ 2010, 2344. 
einen Augenblick die Reaktionen vor, die an einem Samstagnachmittag in einem Ladengeschäft eines internationalen Möbelkonzerns - bis vor nicht allzu langer Zeit $^{11}$ - die Durchsage: „Liebe Eltern, der kleine Djehad möchte im Småland abgeholt werden“ auslösen würde.

Aber auch beim Vornamen ist der Gesetzgeber mittlerweile auf den Plan getreten, und zwar für den Fall, dass der Bürger mit der Reihenfolge seiner Vornamen nicht einverstanden ist. Der Namensträger kann seit dem 1. November 2018 durch Erklärung gegenüber dem Standesamt seine Vornamen neu sortieren. Möglich macht diese Vornamenssortierung nach einer Reform des Personenstandsgesetzes $^{12}$ (PStG) ein neuer $\S 45 a$ PStG $^{13}$, dessen Regelungsumfang wieder einmal zeigt, dass im Namensrecht der Bundesrepublik Deutschland nichts einfach sein kann.

Man sollte sich indessen vom beeindruckenden Normenbestand nicht täuschen lassen. Das deutsche Namensrecht ist weit von einem in sich schlüssigen System entfernt: Das Dickicht ist vor allem durch gesetzgeberische Nachjustierungen für Einzelkonstellationen gewachsen - bereits ein ausreichendes Indiz für einen grundlegenden Reformbedarf. Dabei hat der Gesetzgeber nicht einmal alle namensrechtlichen Fragen geregelt, die praktische Relevanz besitzen: Zu nennen ist neben der eben erwähnten Vornamensbestimmung insbesondere der Schutz des Vertrauens in einen tatsächlich geführten, aber rechtlich unrichtigen Namen, zu dem sich bisher vor allem die verfassungs- und unionsrechtliche Rechtsprechung geäußert hat. ${ }^{14}$ Die Möglichkeit einer Namensersitzung bedarf einer rechtsklaren Regelung, wie nunmehr auch das eingangs erwähnte Eckpunktepapier des Bundesinnenministeriums und des Bundesjustizministeriums fordert. $^{15}$

Diese Einschätzung zum Status quo des deutschen Namensrechts ist freilich nichts Neues: Das Urteil der Fachkreise fällt seit Jahrzehnten vernichtend aus, wie

11 Der namenskundlich neugierige Verfasser bedauert sehr, dass die IKEA Deutschland GmbH \& Co. KG, jedenfalls in den vom Verfasser frequentierten Ladengeschäften, seit einiger Zeit einsame Småland-Kinder nicht mehr öffentlich ausruft, sondern die Eltern mittels vibrierender Transponder benachrichtigt.

12 Personenstandsgesetz vom 19.2.2007, BGBl. 2007 I S. 122.

13 Eingefügt durch das Zweite Gesetz zur Änderung personenstandsrechtlicher Vorschriften vom 17.7.2017, BGBl. 2017 I S. 2522.

14 Siehe die grundlegende Systematisierung von Reinhard Hepting Der Schutz des tatsächlich geführten Namens - Vertrauensschutz in der Rechtsprechung des BVerfG - und etwa auch des EuGH?, StAZ 2013, 1 und StAZ 2013, 34; siehe zuvor auch bereits Günther Grasmann Die internationale Identität des Familiennamens deutscher Mehrstaater nach deutschem IPR, StAZ 1989, 126 (138 ff.).

15 Eckpunkte zur Reform des Namensrechts (Fn. 2) S. 7 unter III. 8. 
ein - freilich unvollständiger - Streifzug durch die Veröffentlichungen einiger Kenner der Materie zeigt:

- „Die Grundlinien des aktuellen Namensrechts sind verworren. Es ist so kompliziert geworden, dass stellenweise der Gesetzgeber selbst den Überblick verloren hat; dabei hat es - trotzdem oder gerade deswegen? - einen Großteil seiner traditionellen Funktionen eingebüßt, ist voller innerer Widersprüche." ${ }^{\text {"16 }}$

- „Die Zeit ist reif dafür, das gesamte Namensrecht, dem eine einheitliche Struktur immer mehr verloren geht, grundlegend zu überarbeiten [...]. Denn dem Bürger kann nicht verständlich gemacht werden, was der Fachmann selbst nicht mehr versteht. “17

- „Das Ehenamensrecht steckt voller Regelungswidersprüche, die nur deswegen nicht offensichtlich sind, weil der Blick durch die Kompliziertheit getrübt wird. Den Durchblick haben nur noch die Standesbeamten, einschlägig befasste Gerichte und Behörden sowie Kommentatoren, also Fachleute."18

- „Im Ergebnis präsentiert sich das geltende Kindesnamensrecht als ein Flickenteppich mit hoch komplizierten und wertungsinkonsistenten Regelungen“, handhabbar „nur noch, weil es von dem hoch qualifizierten, aber auch hoch spezialisierten Berufsstand der Standesbeamten verwaltet wird."19

Auch die Kommentatoren der namensrechtlichen Vorschriften, die um ihre Aufgabe nicht $\mathrm{zu}$ beneiden sind, lassen kaum ein gutes Haar am geltenden Namensrecht. So liest man:

- „Hinzu kommen sachliche Komplizierungen, die durch namensrechtliche Reformen in Verbindung mit einer zunehmenden Dynamisierung und Internationalisierung der Familienverhältnisse bedingt sind, die [zum Teil] aber auch schlicht auf bürokratischem Perfektionismus bei unklaren und widersprüchlichen Zielvorgaben beruhen. Insgesamt steht die Kompliziertheit des Kindesnamensrechts in keinem vernünftigen Verhältnis zur Bedeutung der Thematik. “20

16 Reinhard Hepting Grundlinien des aktuellen Familiennamensrechts, FPR 2002, 115 (120).

17 Heinrich Bornhofen Das geänderte Recht zum Ehenamen und Lebenspartnerschaftsnamen, StAZ 2005, 226 (230).

18 Berthold Gaaz Das deutsche Ehenamensrecht - gestern, heute und morgen, StAZ 2006, 157 (164).

19 Michael Coester Das Kind muss einen Namen haben, Jura 2007, 348 (348 f.).

20 Katharina Hilbig-Lugani in: Staudinger, Kommentar zum Bürgerlichen Gesetzbuch, Bearbeitung 2015, vor § 1616 BGB Rn. 4. 
- „Durch zahlreiche Reformen der letzten Jahre [...] hat die privatrechtliche Regelung inzwischen einen Grad an Komplexität erreicht, welcher außerhalb jedes Verhältnisses zur eigentlichen Bedeutung der Materie im Alltagsleben steht.“'21 „Ob der deutsche Gesetzgeber den Mut zu [...] radikalen Änderungen aufbringen wird, erscheint jedoch sehr fraglich, eher wird es weitergehen mit dem Herumbasteln an der gegenwärtigen Regelung, die immer neue Wertungs- und Regelungswidersprüche hervorbringen wird.“222

- „Dem geltenden Namensrecht ist das Austarieren der hinter den unterschiedlichen Namensfunktionen stehenden Interessen und Grundsätze nur um den Preis eines sehr detaillierten und überkomplizierten Regelwerks gelungen, das übrigens in einer Vielzahl von Fallkonstellationen gleichwohl keine stimmigen Lösungen präsentiert.“23 „Die Bestimmungen zum Ehenamen [...] versuchen in ihrer heutigen Fassung, eine Reihe von - [zum Teil] gegenläufigen - Zielen miteinander in Einklang zu bringen. Das hat zu einer insgesamt höchst komplizierten Regelung geführt, die zudem nicht nur widersprüchliche, sondern [zum Teil] auch sinnwidrige Ergebnisse hervorbringt. ${ }^{24}$

21 Karl August Prinz von Sachsen Gessaphe in: Münchener Kommentar zum Bürgerlichen Gesetzbuch, Band X (8. Aufl. 2020) vor § 1616 BGB Rn. 6.

22 Karl August Prinz von Sachsen Gessaphe in: Münchener Kommentar zum Bürgerlichen Gesetzbuch, Band IX (8. Aufl. 2019) § 1355 BGB Rn. 74.

23 Beate Kienemund in: beck-online.GROSSKOMMENTAR (Stand: 1. 2. 2020) § 1616 BGB Rn. 17.

24 Kienemund (vorige Fn.) § 1355 BGB Rn. 2. 